\title{
Coordinating Distributed Energy Resources and Utility-Scale Battery Energy Storage System for Power Flexibility Provision Under Uncertainty
}

\author{
Mohsen Kalantar-Neyestanaki, Student Member, IEEE, and Rachid Cherkaoui, Senior Member, IEEE
}

\begin{abstract}
Relying on the power flexibility of distributed energy resources (DERs) located in an active distribution network (ADN), this ADN will be able to provide power flexibility to the upperlayer grid at their point of common coupling (PCC). The power flexibility is defined as additional bi-directional active/reactive powers a resource can provide to the grid by adjusting its operating point. In this context, this paper presents a two-stage ADN management method to deliver, at the PCC, the power flexibility that the upper-layer grid operator would request minutes-ahead real-time operation. The first stage updates the power set-points of DERs considering their offer curves as well as the uncertainties stem from the short-term forecast errors of demand and renewable generation profiles. The inter-temporal constraints and losses of the grid are accounted for by exploiting a linearized dynamic optimal power flow model, whereby the first stage is implemented as a linear scenario-based optimization problem. Then, in real-time operation, relying on a linear optimization problem, the second stage adjusts the power flexibility injection of a utility-scale battery energy storage system (ESS) to mitigate the imbalance at the PCC inherent in the abovementioned uncertainties. The performance of the proposed method is tested in the case of a real ADN located in the city of Aigle in southwest of Switzerland.
\end{abstract}

Index Terms-Active distribution network (ADN), ADN management, ancillary services, distributed energy resources (DERs), energy storage system (ESS), linear optimization-based control strategy, power flexibility, stochastic optimization, uncertainty.

\section{INTRODUCTION}

$\mathbf{E}$ nvironmental challenges have lunched an increasing use of renewable energy generation in electrical power systems. However, to guarantee an efficient voltage/frequency regulation in the presence of significant amount of intermittent solar and wind generation, a rapt attention should be devoted to the power flexibility provision issue [1]. In this context, the active and reactive powers flexibility can be defined as additional bi-directional active/reactive powers a given resource can provide to the grid by regulating up or down its operating point. The existing literature offers three categories of methods to deal with power flexibility provision in modern power systems with high penetration of intermittent renewable energy generation. All the three categories set out to facilitate the contribution of different resources and take advantage at

The authors are with Power Systems Group (PWRS), École Polytechnique Fédérale de Lausanne (EPFL), Lausanne, Switzerland (e-mail: most of the available power flexibility of those resources. In this way, they attempt to preserve the security of the grid against increasing uncertainties stem from intermittent renewable generation. The first categories of methods [2]-[5] focuses on the resources connected exclusively to the transmission network and present new approaches for coordinating and managing those resources to thoroughly deploy their power flexibility. The work in [2] proposes a multi-stage method for scheduling the power flexibility of various resources, including thermal units, hydro pumped-storage units and utility-scale batteries, to provide ancillary services to the transmission network. For interconnected power systems with hydro, thermal, wind and solar units, [3] proposes a multi-area power flexibility sharing model. This model takes advantage of the available power flexibility of all areas to deal with uncertainties of solar and wind generation. In [4] a new method is offered to determine the optimal amount of active power flexibility that a transmission system operator (TSO) should book from conventional power plants to deal with uncertainties of demand and intermittent wind generation. Work in [5] quantifies the optimal required active power flexibility of the TSO while considering the uncertainties of wind/solar generation and demand. It tries to minimize the TSO's cost by realizing a balance between the cost associated with booking active power flexibility and the expected cost of energy not served.

On the other hand, tracking the evolution of active distribution networks (ADNs) bears testimony to the fast proliferation of distributed energy resources (DERs) they are hosting [6]. In this emerging architecture, the potential power flexibility of DERs can be exploited to provide a variety of services like congestion management and frequency/voltage regulation not only to the ADN (second category of methods [7]-[13]) but also to the upper-layer grid (third category of methods [14]-[17]). In order to unlock the active/reactive powers flexibility of DERs, tighter collaborations among different actors, including consumers, DER owners, TSOs and distribution system operators (DSOs), should be developed and implemented [14].

In this emerging architecture, the second category of methods [7]-[13] aims to deal with power flexibility provision issue at distribution level by tapping the potential flexibility of DERs. In this regard, a real-time demand-response scheme is proposed in [7] to mitigate the voltage fluctuations in an ADN. In [8], a

mohsen.kalantar@epfl.ch; rachid.cherkaoui@epfl.ch) 


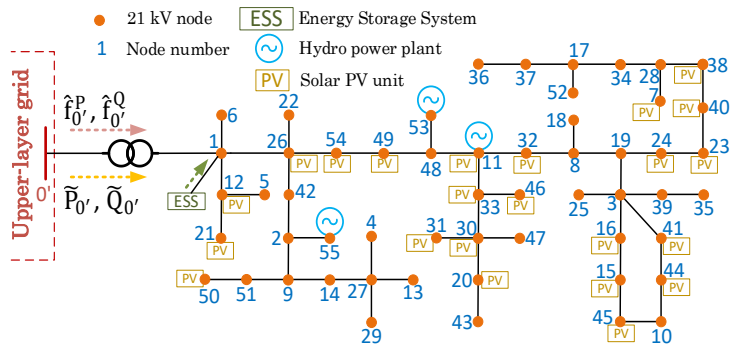

Fig. 1. The single line diagram of an active distribution network located in Aigle (a city in southwest of Switzerland).

stochastic dynamic programming approach is developed to supply the local demand of an isolated nano grid by deploying the power flexibility of energy storage systems and dispatchable generators while considering the uncertainties of PV generation. An optimal power flow management technique is presented in [9] to provide congestion management service in an ADN. The work in [10] proposes a geometric approach to aggregate the power flexibility of thermostatically controlled loads for the purpose of frequency regulation. For day-ahead operation planning of ADNs, dynamic optimal power flow models are designed in [11] and [12] to maximize the exported power to the upper-layer grid while considering the intertemporal constraints of energy storage systems and wind uncertainties. An optimal inverter dispatch framework is presented in [13] that relies on the power flexibility of PVs' inverters to avoid over-voltage in ADNs while considering uncertainties of PV generation.

The third categories of methods [14]-[17] aims to upgrade the top-to-down (from the transmission network to the distribution networks) power flexibility provision mechanism to a bidirectional one. To this end, they set out to unlock and deploy the power flexibility of the DERs located at the distribution level with the purpose of providing it to the upperlayer grid. The works in [15] and [16] introduces optimizationbased methods to estimate the range of active and reactive powers flexibility that an ADN can provide to the upper-layer grid at their point of common coupling (PCC) during each time slot of the next day. Work in [17] takes into account the power flexibility of DERs located at distribution level and introduces a method to quantify the amount of power flexibility that the TSO could require from each DSO.

Although a variety of methods have been developed to unlock and take advantage of the power flexibility of DERs, the existing literature however lacks a framework to answer:

How much should be the active/reactive powers flexibility provided by each DER during the real-time operation in such a way that the ADN can provide, with minimum deviation, the minutes-ahead active/reactive powers flexibility requested by the upper-layer grid at the PCC?

In this respect, for each time slot (e.g. with 15-minute or 1hour duration) of real time operation and a given flexibility request ${ }^{1}$ from the upper-layer grid [15],[16], the ADN operator can determine then deploy the contribution ${ }^{1}$ of each DER in order to fulfill at best that request. However, from one hand, continuous deviations from this target are unavoidable during
Second Stage: At the beginning of each time-interval $\left(\tau_{2}\right)$, the linear optimization problem defined in the second stage of the method is solved. Accordingly, the active/reactive powers flexibility that the ESS should provide throughout the timeinterval is automatically updated.

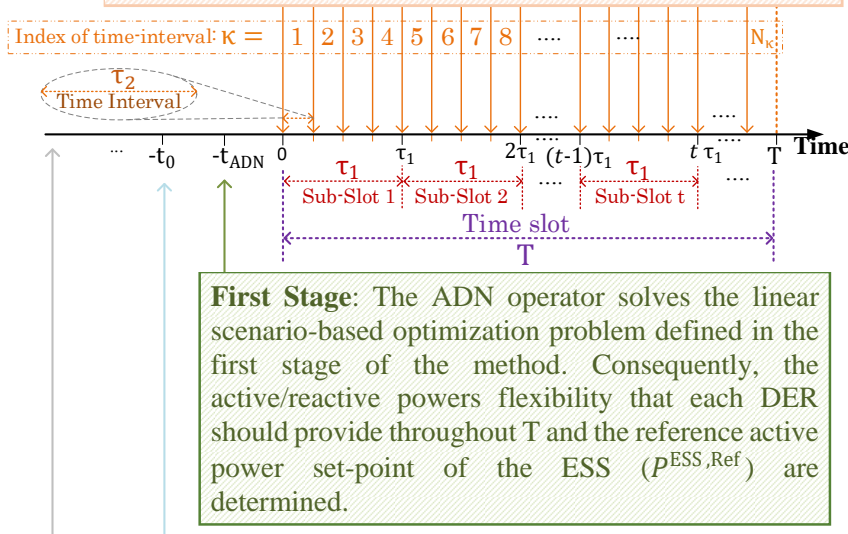

The upper-layer grid operator requests from the ADN operator to provide a specific amount of active/reactive powers flexibility $\left(\hat{\mathrm{f}}_{0}^{\mathrm{P}}\right.$ and $\left.\hat{\mathrm{f}}_{0}^{\mathrm{Q}}\right)$ throughout the coming time slot $(\mathrm{T})$

According to the day-ahead energy market outcome, the scheduled active and reactive powers absorption of the ADN at the PCC, i.e. $\widetilde{\mathrm{P}}_{0}$ and $\widetilde{\mathrm{Q}}_{0}$, are given.

Time offset (in minutes) with respect to the beginning of the time slot. Fig. 2. The timeline of the proposed method for a time slot.

the time slot due to the uncertainties inherent in the demand and renewable generation. From another hand, generally DERs do not have the capability to track automatically and continuously any quantity remotely located such as the power flexibility requested at the PCC. Therefore, in the context of this paper, the ADN is supposed equipped with a battery energy storage system (ESS) connected at the root of the ADN, as illustrated in Fig. 1. This ESS, endowed with appropriate communication and automatic control facilities, aims exclusively at absorbing as much as possible the above-mentioned deviations. Considering this defined task for the ESS, the ESS does not compete with the DERs to provide flexibility to the upper-layer grid.

In view of the above, this paper follows the third category of methods. It proposes a two-stage approach for providing power flexibility from an ADN to its upper layer grid. At each time slot, first the power set-points of the DERs are updated. Then, the ESS is continuously controlled to achieve the target while starting from a specific power set-point value helping to preserve its state of energy (SOE). The details of both stages are presented and illustrated in the next sections.

The rest of the paper is organized as follows: Section II states the problem and contributions of the paper. Sections III and IV present the first and second stages, respectively. Section V demonstrates the proof-of-concept applying the proposed method to the ADN located in the city of Aigle (Switzerland). Finally, Section VI states the main conclusions.

\footnotetext{
${ }^{1}$ Unique value for the whole time slot.
} 


\section{PRoblem Statement And Contributions}

The problem treated in this paper can be described focusing on a single time slot of $\mathrm{T}$ minutes duration as outlined in Fig. 2. This figure embraces three time periods:

- Time slot: It is a time period of T minutes duration. On the basis of the day-ahead energy market outcome, the scheduled operating point of the ADN is known and unique over each time slot (see details in Section III). Moreover, the requested active/reactive powers flexibility of the upper-layer grid operator is a unique value over each time slot.

- Sub-slot: Each time slot consists of a number of sub-slots with duration of $\tau_{1}$ minutes and $t$ is the index of sub-slots. The first stage of the method models the trajectory of uncertainties, i.e. the forecast errors of demand and renewable generation, with time resolution of $\tau_{1}$.

- Time-interval: The whole time slot is split into $\mathrm{N}_{\kappa}$ timeintervals with duration $\tau_{2}$ and $\kappa$ is the index for time-intervals. The second stage of the method is executed at the beginning of each time-interval.

On the basis of the day-ahead energy market outcome, the day-ahead scheduled active and reactive power flows at the PCC of the ADN, i.e. $\widetilde{\mathrm{P}}_{0^{\prime}}$ and $\widetilde{\mathrm{Q}}_{0^{\prime}}$, are known. They are of the time resolution of $\mathrm{T}$ minutes, i.e. a unique operating point for each time slot. Then, $t_{0}$ minutes prior to the beginning of each time slot, the upper-layer grid operator sends to the ADN operator its active/reactive powers flexibility request, i.e. $\hat{\mathrm{f}}_{0^{\prime}}^{\mathrm{P}}$ and $\hat{\mathrm{f}}_{0^{\prime}}^{\mathrm{Q}}$. They are of the time resolution of $\mathrm{T}$ minutes, i.e. unique values for the whole time slot. These two terms form the targeted operating point at the ADN's PCC as:

$\mathrm{P}_{0^{\prime}}^{\text {Target }}=\widetilde{\mathrm{P}}_{0^{\prime}}+\hat{\mathrm{f}}_{0^{\prime}}^{\mathrm{P}}$,

$\mathrm{Q}_{0^{\prime}}^{\text {Target }}=\widetilde{\mathrm{Q}}_{0^{\prime}}+\hat{\mathrm{f}}_{0^{\prime}}^{\mathrm{Q}}$

This targeted operating point is followed thanks to the twostage method introduced in this paper:

Stage1: it is entitled updating the power set-points of DERs and ESS. $\mathrm{t}_{\mathrm{ADN}}$ minutes prior the beginning of each time slot, the first stage determines the new power set-points of the DERs considering their offer curves and accommodating at best the uncertainties stem from the short term forecast errors of demand and renewable generation profiles during the time slot. Simultaneously, regarding these uncertainties, it determines a reference value ${ }^{1}$ for the ESS power set-point. This reference value helps the ESS to achieve its task while guaranteeing an appropriate SOE level during the whole time slot. The determination of all these power set-points is formulated as a linear scenario-based optimization problem where the constraints and the losses of the grid are accounted for thanks to a linearized dynamic power flow model.

Stage 2: it is entitled EES real-time control. Throughout the time slot, all DERs except the ESS follow their power set-points determined in the first stage. Then, to mitigate the deviations from the targeted operating point at PCC which result from the load and generation uncertainties, this stage constructs a linear optimization problem considering the operational constraints of the ESS, whereby it adjusts the reference power set-point of the ESS determined in the first stage.

${ }^{1}$ Unique value for the whole time slot.
In sum, the ADN operator solves both stages of the method considering the known $\mathrm{P}_{0^{\prime}}^{\text {Target }}$ and $\mathrm{Q}_{0^{\prime}}^{\text {Target }}$ defined in (1) and (2). The method is envisaged to bring about three benefits. Firstly, it can provide active/reactive powers flexibility to the upper-layer gird. These powers flexibility can help the upperlayer grid operator to deal with congestion, active power imbalance, overvoltage and undervoltage issues in its grid. Secondly, local provision of powers flexibility can postpone and even decrease the necessity of network reinforcement in the upper-layer grid. Last but not least, it may result in reducing the price of ancillary services thanks to unlocking the power flexibility of new resources, i.e. DERs.

The contributions of the paper can be enumerated as:

- It presents a two-stage ADN management method to procure the power flexibility of DERs with the aim of providing a specific $^{2}$ amount of active/reactive power flexibility to the upper-layer grid at the ADN's PCC. To the best knowledge of the author, it has not yet been addressed in the existing literature.

- It extracts linear equivalent counterparts for nonlinear ESS's/DERs' constraints, DERs' offer curves and objective function, whereby, it offers linear tractable algorithms for the first and second stages of the method.

- Thanks to the extracted linear models, it casts the first stage of the method as a linearized dynamic power flow model. In addition to the technical constraints of the grid and ESS/DERs, this model takes into account the grid's power losses and temporal variations and uncertainties of demand/renewable generation.

- It develops a real-time control strategy for the ESS and mathematically formulates it as a linear optimization problem.

- It offers a novel approach for operating the ESS. This approach divides the net power injection of the ESS into two terms to elaborately preserve the ESS's SOE as well as to take advantage at most of the power flexibility of the ESS, as detailed in III. The results, presented in section V, highlight the advantage of this novel approach.

- It finally illustrates the performance of the proposed method on a real distribution network located in the city of Aigle in southwest of Switzerland.

For the sake of brevity, the rest of the paper focuses on a single time slot except the numerical result section, i.e. section V . Actually, the proposed method can be applied identically to any desired time slot.

\section{First StAGE: Updating THE POWER SET-PoINTS OF DERS AND ESS}

To present the mathematical formulation of the first stage, let $i$ and $j$ be the indices for the nodes excluding the PCC node (i.e. $0^{\prime}$ ); $\mathbb{B}$ the set of nodes excluding $0^{\prime} ; t$ and $t^{\prime}$ the indices for the sub-slots (as depicted in Fig. 2); $\mathbb{T}$ the set of sub-slots belonging to time slot $\mathrm{T}$; $s$ the index for scenarios modeling the forecast errors of demand and renewable generation; $\mathbb{S}$ the set of selected credible scenarios; $l$ the index for the branches; $\mathbb{L}$ the set of branches of the ADN; $k$ the index for the dispatchable distributed generators (DDGs); $\mathbb{D D G}$ the set of DDGs

\footnotetext{
${ }^{2}$ Requested by the upper-layer grid operator.
} 
connected to node $i ; \mathbb{D D G}$ the set of DDGs located in the ADN; $h$ the index for the renewable distributed generators (RDGs); $\mathbb{R D} G_{i}$ the set of RDGs connected to node $i ; \mathbb{R D G}$ the set of RDGs located in the ADN; operator $|$.$| denotes the$ absolute values of its argument. Without loss of the problem's generality, it is here assumed that:

- each DDG can take only a single power set-point over the whole time slot $\mathrm{T}$;

- DDGs can provide active and reactive powers flexibility, i.e. $f_{k}^{\mathrm{DDG}, \mathrm{P}}$ and $f_{k}^{\mathrm{DDG}, \mathrm{Q}}$, in addition to their scheduled ${ }^{1}$ active/reactive power injections, i.e. $\widetilde{P}_{k}^{\text {DDG }}$ and $\widetilde{Q}_{k}^{\text {DDG }}$;

- the trajectory of the forecasted active power injection of the RDGs, i.e. $\widehat{P}_{h t}^{\mathrm{RDG}}$, are considered with time resolution of $\tau_{1}$;

- the scheduled ${ }^{1}$ reactive power injection of RDGs, i.e. $\widetilde{\mathrm{Q}}_{h}^{\mathrm{RDG}}$, are assumed to be 0 ;

- RDGs are sources of active power uncertainties, i.e. $\Delta \mathrm{P}_{h t s}^{\mathrm{RDG}}$. Thus, they might deviate from their forecasted active power injection $\widehat{\mathrm{P}}_{h t}^{\mathrm{RDG}}$. However, they can provide reactive power flexibility, i.e. $f_{h}^{\mathrm{RDG}, \mathrm{Q}}$ (unique value for the whole time slot);

- the trajectory of the forecasted active/reactive power absorption of loads, i.e. $\widehat{P}_{i t}^{\mathrm{D}}$ and $\widehat{\mathrm{Q}}_{i t}^{\mathrm{D}}$, are considered with time resolution of $\tau_{1}$;

- loads are sources of active/reactive power uncertainties, i.e. $\Delta \mathrm{P}_{i t s}^{\mathrm{D}} / \Delta \mathrm{Q}_{i t s}^{\mathrm{D}}$. Thus, they might deviate from their forecasted active/reactive power absorption, i.e. $\widehat{\mathrm{P}}_{i t}^{\mathrm{D}} / \widehat{\mathrm{Q}}_{i t}^{\mathrm{D}}$;

- the forecast errors of renewable generation and demand, i.e. $\Delta \mathrm{P}_{\text {hts }}^{\mathrm{RDG}}, \Delta \mathrm{P}_{i t s}^{\mathrm{D}}$ and $\Delta \mathrm{Q}_{i t s}^{\mathrm{D}}$ are modeled through a set of scenarios with time resolution of $\tau_{1}$;

- the scheduled ${ }^{1}$ active/reactive power injections of the ESS (connected at the root of the ADN as shown in Fig. 1) are 0;

- The rationale behind modeling the ESS in the first stage is to preserve its SOE, i.e. empower the ESS to provide successfully flexibility during the second stage. Since the ESS's SOE is directly affected only by its active power flexibility provision, the first stage assumes the reactive power flexibility provision of the ESS equal to zero. Therefore, the first stage determines only the net active power injection of the ESS, i.e. $P_{t s}^{\mathrm{ESS}, \mathrm{Net}}$, for each sub-slot $t$ and scenario $s . P_{t s}^{\mathrm{ESS}, \mathrm{Net}}$ consists in the sum of two terms:

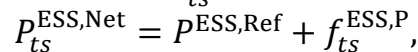

$P^{\mathrm{ESS}, \text { Ref }}$ indicates the reference power set-point of the ESS over the whole time slot. It is positive or negative active power that the ESS should exchange over the whole time slot to restore an adequate SOE. For each individual scenario s, $f_{t s}^{\mathrm{ESS}, \mathrm{P}}$ indicates the final adjustment of the ESS's set-point over sub-slot $t$ that is expected to be accomplished by the ADN operator during the second stage.

In sum, it is worth highlighting that the scheduled/forecasted power set-points of DERs, i.e. $\widetilde{\mathrm{P}}_{k}^{\mathrm{DDG}}, \widetilde{\mathrm{Q}}_{k}^{\mathrm{DDG}}, \widehat{\mathrm{P}}_{h t}^{\mathrm{RDG}}$ and $\widetilde{\mathrm{Q}}_{h}^{\mathrm{RDG}}$ are known, i.e. parameter.

\section{A. Objective Function}

The objective function is designed to satisfy as much as possible the targeted power flow at the PCC with minimum cost. It can be mathematically formulated as: $\min _{\xi} C_{0^{\prime}}^{\mathrm{Imb}}+C^{\mathrm{ESS}}+\sum_{k \in \mathbb{D} \mathbb{D} G}\left[C_{k}^{\mathrm{DDG}, \mathrm{P}+}+C_{k}^{\mathrm{DDG}, \mathrm{P}-}\right]$

where $\xi$ indicates the set of optimization variables as: $\xi=\left\{f_{k}^{\mathrm{DDG}, \mathrm{P}}, f_{k}^{\mathrm{DDG}, \mathrm{Q}}, f_{h}^{\mathrm{RDG}, \mathrm{Q}}, P_{t s}^{\mathrm{ESS}, \mathrm{Net}}, P^{\mathrm{ESS}, \mathrm{Ref}}, f_{t s}^{\mathrm{ESS}, \mathrm{P}}\right.$,

$$
\left.P_{0^{\prime} t s}, Q_{0^{\prime} t s}, V_{i t s}, I_{l t s}^{\text {Real }}, I_{l t s}^{\text {Imag }}\right\},
$$

the undefined variables of (5) will be introduced in the rest of the section beside their corresponding constraints.

The objective function (4) includes three parts:

\section{1) Penalizing the Imbalance of the $A D N$ at the PCC}

The active and reactive power imbalances of the ADN at the PCC are (for all $t$ in $\mathbb{T}$ and $s$ in $\mathbb{S}$ ):

$P_{0^{\prime} t s}^{\mathrm{Imb}}=P_{0^{\prime} t s}-\mathrm{P}_{0^{\prime}}^{\text {Target }}$,

$Q_{0^{\prime} t s}^{\text {Imb }}=Q_{0^{\prime} t s}-Q_{0^{\prime}}^{\text {Target }}$,

where $P_{0^{\prime} t s}$ and $Q_{0^{\prime} t s}$ are respectively active/reactive power flow at the PCC, as detailed in (32) and (33). The proposed method tries to follow the targeted power flow at the PCC with minimum deviations throughout the time slot. Thus, it assigns a virtual cost to the active/reactive power imbalances as:

$C_{0^{\prime}}^{\mathrm{Imb}}=\pi_{0^{\prime}}^{\mathrm{Imb}, \mathrm{P}} \sum_{s \in \mathbb{S}} \sum_{t \in \mathbb{T}}\left|P_{0^{\prime} t s}^{\mathrm{Imb}}\right|+\pi_{0^{\prime}}^{\mathrm{Imb}, Q} \sum_{s \in \mathbb{S}} \sum_{t \in \mathbb{T}}\left|Q_{0^{\prime} t s}^{\mathrm{Imb}}\right|$,

where $\pi_{0^{\prime}}^{\mathrm{Imb}, \mathrm{P}}$ and $\pi_{0^{\prime}}^{\mathrm{Imb}, \mathrm{Q}}$ are virtual large weighting coefficients. Minimizing the nonlinear term (8), i.e. the first part of (4), has a linear equivalent counterpart as:

$\min \pi_{0^{\prime}}^{\mathrm{Imb}, \mathrm{P}} \sum_{s \in \mathbb{S}} \sum_{t \in \mathbb{T}} \gamma_{0^{\prime} t s}^{\mathrm{Imb}, \mathrm{P}}+\pi_{0^{\prime}}^{\mathrm{Imb}, \mathrm{Q}} \sum_{s \in \mathbb{S}} \sum_{t \in \mathbb{T}} \gamma_{0^{\prime} t s}^{\mathrm{Imb}, \mathrm{Q}}$,

subject to (for all $t$ in $\mathbb{T}$ and $s$ in $\mathbb{S}$ ):

$-\gamma_{0^{\prime} t s}^{\operatorname{Imb}, \mathrm{P}} \leq P_{0^{\prime} t s}^{\mathrm{Imb}} \leq \gamma_{0^{\prime} t s}^{\mathrm{Imb}, \mathrm{P}}$,

$-\gamma_{0^{\prime} t s}^{\operatorname{Imb}, \mathrm{Q}} \leq Q_{0^{\prime} t s}^{\operatorname{Imb}} \leq \gamma_{0^{\prime} t s}^{\operatorname{Imb}, \mathrm{Q}}$,

where $\gamma_{0^{\prime} t s}^{\text {Imb,P }}$ and $\gamma_{0^{\prime} t s}^{\text {Imb,Q }}$ are non-negative auxiliary variables.

\section{2) Penalizing the deployed flexibility from the ESS}

To keep at most the capability of the ESS for providing both upward and downward active power flexibility, the first stage aims to maintain the SOE close to the middle of its maximum and minimum allowed values by defining the targeted active power set-point of the ESS, i.e. $P^{\text {ESS,Target }}$, as:

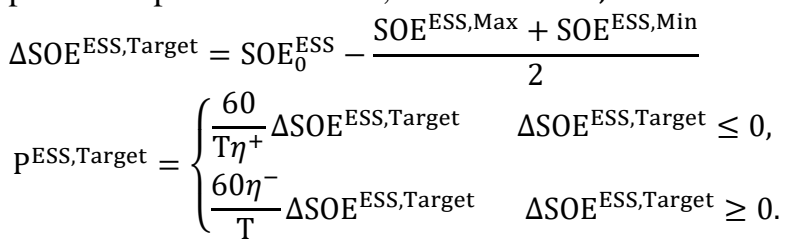

where $\mathrm{SOE}_{0}^{\mathrm{ESS}}$ is initial SOE, $\mathrm{SOE}^{\mathrm{ESS}, \mathrm{Max}}$ and $\mathrm{SOE}^{\mathrm{ESS}, \mathrm{Min}}$ are respectively maximum and minimum limit of the ESS's SOE. $\triangle \mathrm{SOE}^{\mathrm{ESS} \text {, Target }}$ is the difference between the initial SOE and the average of SOE ${ }^{\mathrm{ESS}, \mathrm{Min}}$ and $\mathrm{SOE}^{\mathrm{ESS}, \mathrm{Max}} . \eta^{+}$and $\eta^{-}$are the charging and discharging efficiency of the ESS, respectively. Multiplier $\frac{1}{T / 60}$ converts the duration of the time slot from minute to hour.

The first stage tries to minimize the required active power flexibility from the ESS during the second stage, i.e. $f_{t s}^{\mathrm{ESS}, \mathrm{P}}$, and

\footnotetext{
${ }^{1}$ which are known from the day-ahead energy market outcome.
} 
to keep $P^{\mathrm{ESS} \text {, Ref }}$ close to the targeted value $\mathrm{P}^{\mathrm{ESS} \text {,Target }}$. These two goals are achieved by defining the virtual cost:

$$
\begin{aligned}
& C^{\mathrm{ESS}}=\pi^{\mathrm{ESS}, \mathrm{P}} \sum_{s \in \mathbb{S}} \sum_{t \in \mathbb{T}}\left|f_{t s}^{\mathrm{ESS}, \mathrm{P}}\right|+ \\
& +\pi^{\mathrm{ESS}, \mathrm{SOE}}\left|P^{\mathrm{ESS}, \mathrm{Ref}}-\mathrm{P}^{\mathrm{ESS}, \text { Target }}\right|+\sum_{s \in \mathbb{S}} \sum_{t \in \mathbb{T}} \gamma_{t s}^{\mathrm{ESS}, \text { Pnet }},
\end{aligned}
$$

where $\pi^{\mathrm{ESS}, \mathrm{P}}$ and $\pi^{\mathrm{ESS}, \mathrm{SOE}}$ are virtual weighting coefficients. The third term, i.e. $\gamma_{t s}^{\text {ESS,Pnet }}$, is an auxiliary variable defined to support the linear model of the evolution of the SOE over time, as detailed in (16), (19) and (44).

It is noteworthy that the ESS is the sole resource that is equipped with appropriate communication and automatic control facilities. In other words, the ESS is the sole resource that the $\mathrm{ADN}$ operator can changes its power set-point during the real-time operation, i.e. the second stage. Accordingly, during the real-time operation where the exact amount of uncertainties is unfolded, the ADN operator can only deploy the power flexibility of the ESS to mitigate the impact of the uncertainties on the active/reactive power imbalance at the PCC. Considering this fact, the method fully dedicates the ESS to the second stage, i.e. real-time operation. Otherwise, the ESS's free capacity for canceling out the impact of the uncertainties during the real-time operation will be jeopardized.

To implement the above-mention set-up, the power flexibility $f_{t s}^{\mathrm{ESS}, \mathrm{P}}$ is prevented to compete with the power flexibilities from the DDGs as a contribution to the power flexibility request of the upper-layer grid operator. It implies that the weighting coefficient $\pi^{\mathrm{ESS}, \mathrm{P}}$ must be large in comparison with the DDG flexibility offer prices. In addition, the average of $f_{t s}^{\text {ESS,P }}$ over all scenarios is enforced to be zero, thereby:

$\frac{1}{\mathrm{~N}_{s}} \sum_{s \in \mathbb{S}} f_{t s}^{\mathrm{ESS}, \mathrm{P}}=0 \quad \forall t \in \mathbb{T}$,

where $\mathrm{N}_{S}$ is the number of scenarios belonging to $\mathbb{S}$. This constraint helps to avoid a constant offset of $f_{t s}^{\mathrm{ESS}, \mathrm{P}}$ all along the whole time slot. It is notable that (3), (14) and (15) enforce the average of the ESS's net provided active power flexibility, i.e. $P_{t s}^{\mathrm{ESS}, \mathrm{Net}}$, over all scenarios to be a constant value equal to $\mathrm{P}^{\mathrm{ESS} \text {,Target }}$. Thus, its SOE is expected to remain close to the middle.

Minimizing the nonlinear term (14), i.e. the second part of (4), has a linear equivalent counterpart as:

$$
\begin{aligned}
\min \pi^{\mathrm{ESS}, \mathrm{P}} \sum_{s \in \mathbb{S}} \sum_{t \in \mathbb{T}} \gamma_{t s}^{\mathrm{ESS}, \mathrm{P}}+\pi^{\mathrm{ESS}, \mathrm{SOE}} \gamma^{\mathrm{ESS}, \mathrm{SOE}}+ \\
+\sum_{s \in \mathbb{S}} \sum_{t \in \mathbb{T}} \gamma_{t s}^{\mathrm{ESS}, \text { Pnet }},
\end{aligned}
$$

subject to (for all $t$ in $\mathbb{T}$ and $s$ in $\mathbb{S}$ ):

$-\gamma_{t s}^{\mathrm{ESS}, \mathrm{P}} \leq f_{t s}^{\mathrm{ESS}, \mathrm{P}} \leq \gamma_{t s}^{\mathrm{ESS}, \mathrm{P}}$

$-\gamma^{\mathrm{ESS}, \mathrm{SOE}} \leq P^{\mathrm{ESS}, \mathrm{Ref}}-\mathrm{P}^{\mathrm{ESS}, \text { Target }} \leq \gamma^{\mathrm{ESS}, \mathrm{SOE}}$,

$-\gamma_{t s}^{\text {ESS,Pnet }} \leq P_{t s}^{\text {ESS,Net }} \leq \gamma_{t s}^{\text {ESS,Pnet }}$,

where $\gamma_{t s}^{\mathrm{ESS}, \mathrm{P}}, \gamma^{\mathrm{ESS}, \mathrm{SOE}}$ and $\gamma_{t s}^{\mathrm{ESS}, \mathrm{Pne}}$ variables.

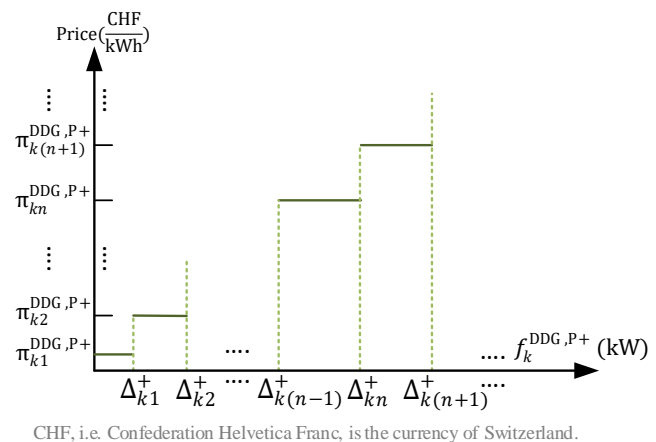

Fig. 3. Offer curve of DDG $k$ for upward active power flexibility provision.

\section{3) Cost of Flexibility Procurement from DERs}

The provided active power flexibility of DDG $k$, i.e. $f_{k}^{\text {DDG,P }}$, can be divided into two non-negative components called upward, i.e. $f_{k}^{\mathrm{DDG}, \mathrm{P}+}$, and downward, i.e. $f_{k}^{\mathrm{DDG}, \mathrm{P}-}$, as:

$f_{k}^{\mathrm{DDG}, \mathrm{P}}=f_{k}^{\mathrm{DDG}, \mathrm{P}+}-f_{k}^{\mathrm{DDG}, \mathrm{P}-} \quad \forall k \in \mathbb{D} \mathbb{D} \mathbb{G}$,

Each DDG offers its prices for the upward and downward active power flexibility provision to the ADN operator through two separate offer curves. For instance, the offer curve of DDG $k$ for its upward active power flexibility is shown in Fig. 3 where $n$ is the index for the offered blocks of DDG $k ; \pi_{k n}^{\mathrm{DDG}, \mathrm{P}+}$ is the price over the $n$th block; $\Delta_{k(n-1)}^{+}$and $\Delta_{k n}^{+}$are the beginning and the end of the $n$th block.

The area under the offer curve defines $C_{k}^{\mathrm{DDG}, \mathrm{P}+}$ indicating the cost that the ADN operator pays to DDG $k$ to procure $f_{k}^{\mathrm{DDG}, \mathrm{P}+}$ for one hour. $C_{k}^{\mathrm{DDG}, \mathrm{P}+}$ is a piece-wise linear function of $f_{k}^{\mathrm{DDG}, \mathrm{P}+}$ :

$C_{k}^{\mathrm{DDG}, \mathrm{P}+}=\pi_{k n}^{\mathrm{DDG}, \mathrm{P}+} f_{k}^{\mathrm{DDG}, \mathrm{P}+}-\pi_{k n}^{\mathrm{DDG}, \mathrm{P}+} \Delta_{k(n-1)}^{+}+$

$$
+\sum_{n^{\prime}=1}^{n-1} \pi_{k n^{\prime}}^{\mathrm{DDG}, \mathrm{P}+} \Delta_{k n^{\prime}}^{+}, \quad \Delta_{k(n-1)}^{+} \leq f_{k}^{\mathrm{DDG}, \mathrm{P}+} \leq \Delta_{k n}^{+}, \quad \forall n,
$$

where $n^{\prime}$ is the index for the offered blocks of DDG $k$. In the same way, $C_{k}^{\mathrm{DDG}, \mathrm{P}-}$ can be calculated as:

$C_{k}^{\mathrm{DDG}, \mathrm{P}-}=\pi_{k n}^{\mathrm{DDG}, \mathrm{P}-} f_{k}^{\mathrm{DDG}, \mathrm{P}-}-\pi_{k n}^{\mathrm{DDG}, \mathrm{P}-} \Delta_{k(n-1)}^{-}+$

$+\sum_{n^{\prime}=1}^{n-1} \pi_{k n^{\prime}}^{\mathrm{DDG}, \mathrm{P}-} \Delta_{k n^{\prime}}^{-}, \quad \Delta_{k(n-1)}^{-} \leq f_{k}^{\mathrm{DDG}, \mathrm{P}-} \leq \Delta_{k n}^{-}, \quad \forall n$.

The third part of the objective function (4) aims at minimizing $C_{k}^{\mathrm{DDG}, \mathrm{P}+}$ and $C_{k}^{\mathrm{DDG}, \mathrm{P}-}$ which are positive increasing functions. Thus, the optimum solution entails that only one of the two variables $f_{k}^{\mathrm{DDG}, \mathrm{P}+}$ and $f_{k}^{\mathrm{DDG}, \mathrm{P}-}$ can be nonzero. In other words, $f_{k}^{\mathrm{DDG}, \mathrm{P}+}$ and $f_{k}^{\mathrm{DDG}, \mathrm{P}-}$ are complementary variables and DDG $k$ can provide either upward or downward active power flexibility (not both simultaneously).

The third part of (4), i.e. the sum of (21) and (22), is a piecewise linear function. However, it has a linear equivalent as:

$\min \sum_{k \in \mathbb{D} \mathbb{D} G}\left[\gamma_{k}^{\mathrm{DDG}, \mathrm{P}+}+\gamma_{k}^{\mathrm{DDG}, \mathrm{P}-}\right]$,

$\frac{\mathrm{T}}{60} C_{k}^{\mathrm{DDG}, \mathrm{P}+} \leq \gamma_{k}^{\mathrm{DDG}, \mathrm{P}+} \quad \forall k \in \mathbb{D} \mathbb{D} G$,

$\frac{\mathrm{T}}{60} C_{k}^{\mathrm{DDG}, \mathrm{P}-} \leq \gamma_{k}^{\mathrm{DDG}, \mathrm{P}-} \quad \forall k \in \mathbb{D D G}$,

where multiplier $\frac{T}{60}$ converts the cost during an hour, i.e. 


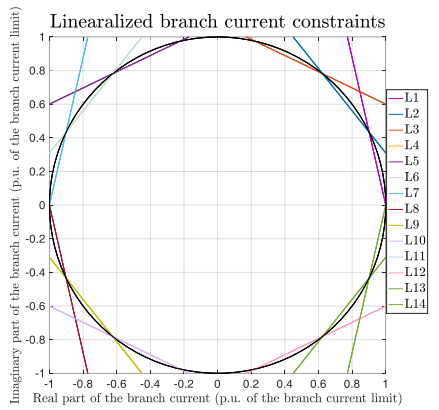

Fig. 4. Linearized ampacity constraint of a branch.

$C_{k}^{\mathrm{DDG}, \mathrm{P}+}$ and $C_{k}^{\mathrm{DDG}, \mathrm{P}-}$, to the cost during $\mathrm{T}$ minutes of a time slot; $\gamma_{k}^{\mathrm{DDG}, \mathrm{P}+}$ and $\gamma_{k}^{\mathrm{DDG}, \mathrm{P}-}$ are non-negative auxiliary variables.

In regard to the reactive power flexibility, it is assumed that the ADN operator has long-term contracts with DERs and can procure their reactive power flexibility without any additional cost. Thus, the reactive power flexibility procurement from DDGs and RDGs causes no cost.

\section{B. Modeling the Constraints of the Grid, DERs and ESS}

This section constructs a scenario-based dynamic power flow model to model the technical constraints of the grid, DERs and the ESS. This model takes into account the grid's power losses and temporal variations and uncertainties of demand/renewable generation throughout the time slot. This model is extracted from the linearized power flow model presented in [18]. It expresses the power flows at the PCC, the voltage magnitudes of all the nodes and the current phasors of all the branches as linear functions of the nodal injections. As detailed in [18], the coefficients of the linear functions can be calculated in close form from the grid admittance matrix, the voltage magnitude at the PCC (slack node) and grid operating point.

The nodal active/reactive power injections at node $i$ during sub-slot $t$ and scenario $s$, i.e. $P_{i t s}$ and $Q_{i t s}$, consists of two terms:

$P_{i t s}=\widehat{\mathrm{P}}_{i t}+\Delta P_{i t s}$

$Q_{i t s}=\widehat{\mathrm{Q}}_{i t}+\Delta Q_{i t s}$.

1t $/ \mathrm{Q}_{\text {it }}$ : It indicates the forecasted nodal active/reactive power injections, i.e., combination of the forecasted and/or scheduled values (for all nodes $i$ in $\mathbb{B}$ and $t$ in $\mathbb{T}$ ):

$$
\begin{aligned}
& \widehat{\mathrm{P}}_{i t}=-\widehat{\mathrm{P}}_{i t}^{\mathrm{D}}+\sum_{k \in \mathbb{D D D} \mathbb{G}_{i}} \widetilde{\mathrm{P}}_{k}^{\mathrm{DDG}}+\sum_{h \in \mathbb{R} \mathbb{B} \mathbb{G}_{i}} \widehat{\mathrm{P}}_{h t}^{\mathrm{RDG}} \\
& \widehat{\mathrm{Q}}_{i t}=-\widehat{\mathrm{Q}}_{i t}^{\mathrm{D}}+\sum_{k \in \mathbb{D D} \mathbb{G}_{i}} \widetilde{\mathrm{Q}}_{k}^{\mathrm{DDG}}+\sum_{h \in \mathbb{R} \mathbb{D} \mathbb{G}_{i}} \widetilde{\mathrm{Q}}_{h}^{\mathrm{RDG}}
\end{aligned}
$$

2) $\Delta P_{i t s} / \Delta Q_{i t s}:$ It indicates the nodal active/reactive power deviations from $\widehat{\mathrm{P}}_{i t} / \widehat{\mathrm{Q}}_{i t}$ (for all nodes $i$ in $\mathbb{B}, t$ in $\mathbb{T}$, and $s$ in $\mathbb{S}$ ):

$$
\begin{aligned}
& \Delta P_{i t s}=-\Delta \mathrm{P}_{i t s}^{\mathrm{D}}+\sum_{k \in \mathbb{D D D} \mathbb{G}_{i}} f_{k}^{\mathrm{DDG}, \mathrm{P}}+\sum_{h \in \mathbb{R} \mathbb{B} \mathbb{G}_{i}} \Delta \mathrm{P}_{h t s}^{\mathrm{RDG}}+ \\
& \Delta Q_{i t s}=-\Delta \mathrm{Q}_{i t s}^{\mathrm{D}}+\sum_{k \in \mathbb{D} \mathbb{B} \mathbb{G}_{i}} f_{k}^{\mathrm{DDG}, \mathrm{Q}}+\sum_{h \in \mathbb{R} \mathbb{D} \mathbb{G}_{i}}^{P_{t s}^{\mathrm{ESS}, \mathrm{Net}}} f_{h}^{\mathrm{RDG}, \mathrm{Q}}
\end{aligned}
$$

where $a_{E S S}$ is a constant parameter equal to 1 if $i=1$ and 0 otherwise.

\section{1) The Active/Reactive Power Flow at the PCC}

The active power flow at the PCC, for all $t$ in $\mathbb{T}$ and $s$ in $\mathbb{S}$, can be expressed as a linear function of $\Delta P_{i t s}$ and $\Delta Q_{i t s}$ as:

$P_{0^{\prime} t s}=\mathbf{P}_{t}^{0}+\sum_{i \in \mathbb{B}}\left(\mathbf{P}_{i t}^{\mathrm{P}} \Delta P_{i t s}+\mathbf{P}_{i t}^{\mathrm{Q}} \Delta Q_{i t s}\right)$,

where $\mathbf{P}_{t}^{0}, \mathbf{P}_{i t}^{\mathrm{P}}$ and $\mathbf{P}_{i t}^{\mathrm{Q}}$ are constant coefficeints.

The reactive power flow at the PCC, for all $t$ in $\mathbb{T}$ and $s$ in $\mathbb{S}$, can be expressed as a linear function of $\Delta P_{i t s}$ and $\Delta Q_{i t s}$ as:

$Q_{0^{\prime} t s}=\mathbf{Q}_{t}^{0}+\sum_{i \in \mathbb{B}}\left(\mathbf{Q}_{i t}^{\mathrm{P}} \Delta P_{i t s}+\mathbf{Q}_{i t}^{\mathrm{Q}} \Delta Q_{i t s}\right)$.

where $\mathbf{Q}_{t}^{0}, \mathbf{Q}_{i t}^{\mathrm{P}}$ and $\mathbf{Q}_{i t}^{\mathrm{Q}}$ are constant coefficients.

\section{2) Voltage Magnitude Constraint of the ADN nodes}

The voltage magnitude of node $i$, for all $t$ in $\mathbb{T}$ and $s$ in $\mathbb{S}$, can be expressed as a linear function with constant coefficients $\mathbf{V}_{i t}^{0}, \mathbf{V}_{i j t}^{\mathrm{P}}$ and $\mathbf{V}_{i j t}^{\mathrm{Q}}$ :

$V_{i t s}=\mathbf{V}_{i t}^{0}+\sum_{j \in \mathbb{B}}\left(\mathbf{V}_{i j t}^{\mathrm{P}} \Delta P_{j t s}+\mathbf{V}_{i j t}^{\mathrm{Q}} \Delta Q_{j t s}\right)$,

whereby the nodal voltage magnitude limits can be linearly expressed as:

$\mathrm{V}_{i}^{\mathrm{Min}} \leq V_{i t s} \leq \mathrm{V}_{i}^{\mathrm{Max}}$

$\forall i \in \mathbb{B}, \forall t \in \mathbb{T}, \forall s \in \mathbb{S}, \quad(35)$ where $\mathrm{V}_{i}^{\mathrm{Min}}$ and $\mathrm{V}_{i}^{\mathrm{Max}}$ are the minimum and maximum voltage magnitude limit of node $i$.

\section{3) Current Flow Constraint of the ADN Branches}

The real (respectively imaginary) part of the current phasor of branch $l$, for all $t$ in $\mathbb{T}$ and $s$ in $\mathbb{S}$, can be expressed as a linear function with constant coefficients $\mathbf{I}_{l t}^{0, \text { Real }}, \mathbf{I}_{\text {lit }}^{\mathrm{P}, \text { Real }}$ and $\mathbf{I}_{\text {lit }}^{\mathrm{Q} \text {, Real }}$ (respectively $\mathbf{I}_{l t}^{\text {O,Imag }}, \mathbf{I}_{\text {lit }}^{\text {P,Imag }}$ and $\mathbf{I}_{l i t}^{\text {QImag }}$ ):

$I_{l t s}^{\text {Real }}=\mathbf{I}_{l t}^{0, \text { Real }}+\sum_{i \in \mathbb{B}}\left(\mathbf{I}_{\text {lit }}^{\text {P,Real }} \Delta P_{i t s}+\mathbf{I}_{\text {lit }}^{\text {, Real }} \Delta Q_{i t s}\right)$,

$I_{l t s}^{\text {Imag }}=\mathbf{I}_{l t}^{0, I m a g}+\sum_{i \in \mathbb{B}}\left(\mathbf{I}_{\text {lit }}^{\mathrm{P}, \text { Imag }} \Delta P_{i t s}+\mathbf{I}_{\text {lit }}^{\mathrm{Q}, \text { Imag }} \Delta Q_{i t s}\right)$.

Relying on (36) and (37), the ampacity constraint of branch $l^{1}$ can be expressed as:

$I_{l t s}^{\mathrm{Real}^{2}}+I_{l t s}^{\mathrm{Imag}^{2}} \leq \mathrm{I}_{l}^{\mathrm{Max}^{2}}$

$\forall l \in \mathbb{L}, \forall t \in \mathbb{T}, \forall s \in \mathbb{S}, \quad(38)$

where $\mathrm{I}_{l}^{\mathrm{Max}}$ is the maximum current flow limit of branch $l$. As shown in Fig. 4, the nonlinear constraint (38) can be approximated as a set of linear constraints with constant coefficients $\mathbf{A}_{f l}^{\text {Real }}, \mathbf{A}_{f l}^{\text {Imag }}$ and $\mathbf{A}_{f l}^{0}$ :

$\mathbf{A}_{f l}^{\text {Real }} I_{l t s}^{\text {Real }}+\mathbf{A}_{f l}^{\text {Imag }} I_{l t s}^{\text {Imag }} \leq \mathbf{A}_{f l}^{0}$

$\forall l \in \mathbb{L}, \forall f \in \mathbb{A}_{l}, \forall t \in \mathbb{T}, \forall s \in \mathbb{S}$,

where $\mathbb{A}_{l}$ is the set of linear constraints modeling the nonlinear ampacity constraint of branch $l$ and $f$ is the index for those linear constraints belonging to $\mathbb{A}_{l}$.

\section{4) Modeling the Capability Area of DERs}

To take advantage at most of the total available power flexibility of DERs, the proposed method considers the real

\footnotetext{
${ }^{1}$ The maximum current flow limit of branch $l$ is modeled for its both sending and receiving ends.
} 

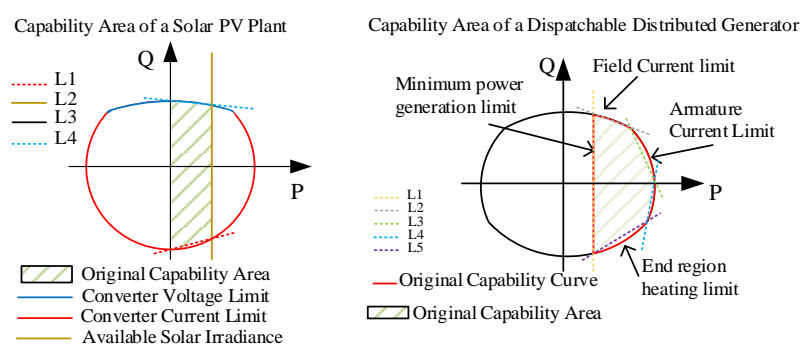

Fig. 5. Linearized Capability area of a solar-PV/dispatchable generator.

nonlinear capability area of each DER and approximates it by using a set of linear boundaries, as exemplified in Fig. 5. In this way, the capability area of DDG $k$ can be expressed as a set of linear constraints with constant coefficients $\mathbf{D}_{k m}^{0}, \mathbf{D}_{k m}^{\mathrm{P}}$ and $\mathbf{D}_{k m}^{\mathrm{Q}}$ as:

$\mathbf{D}_{k m}^{0}+\mathbf{D}_{k m}^{\mathrm{P}} f_{k}^{\mathrm{DDG}, \mathrm{P}}+\mathbf{D}_{k m}^{\mathrm{Q}} f_{k}^{\mathrm{DDG}, \mathrm{Q}} \leq 0$

$\forall k \in \mathbb{D} \mathbb{D} G, \forall m \in \mathbb{A}_{k}, \quad(40)$

and the capability limits of RDG $h$ can be expressed as a set of linear constraints with constant coefficients $\mathbf{R}_{h m t s}^{0}, \mathbf{R}_{h m t s}^{\mathrm{P}}$ and $\mathbf{R}_{h m t s}^{\mathbf{Q}}$ as:

$\mathbf{R}_{h m t s}^{0}+\mathbf{R}_{h m t s}^{\mathrm{P}} \Delta \mathrm{P}_{h t s}^{\mathrm{RDG}}+\mathbf{R}_{h m t s}^{\mathrm{Q}} f_{h}^{\mathrm{RDG}, \mathrm{Q}} \leq 0$

$\forall h \in \mathbb{R} \mathbb{D} G, \forall m \in \mathbb{A}_{h}, \forall t \in \mathbb{T}, \forall s \in \mathbb{S}$, (41)

where $\mathbb{A}_{k}$ and $\mathbb{A}_{h}$ are the sets of linear constraints modeling the nonlinear capability area of DDG $k$ and RDG $h$, respectively. $m$ is the index for the linear constraints belonging to $\mathbb{A}_{k}$ or $\mathbb{A}_{h}$.

\section{5) Modeling the Constraints of the Battery ESS}

The power and energy limits of the ESS can be expressed as: $-S^{\mathrm{ESS}, \text { Max }} \leq P_{t s}^{\mathrm{ESS}, \mathrm{Net}} \leq \mathrm{S}^{\mathrm{ESS}, \mathrm{Max}} \quad \forall t \in \mathbb{T}, \forall s \in \mathbb{S}, \quad$ (42) $\mathrm{SOE}^{\mathrm{ESS}, \mathrm{Min}} \leq S O E_{t s}^{\mathrm{ESS}} \leq \mathrm{SOE}^{\mathrm{ESS}, \mathrm{Max}} \forall t \in \mathbb{T}, \forall s \in \mathbb{S}$, (43) where $S^{\mathrm{ESS}, \mathrm{Max}}$ is the ESS's rated power limit; $S O E_{t s}^{\mathrm{ESS}}$ is the ESS's SOE over sub-slot $t$ and scenario $s$. The evolution of $S O E_{t s}^{\mathrm{ESS}}$ over time can be expressed as a linear function of $P_{t s}^{\mathrm{ESS}, \mathrm{Net}}$ and $\gamma_{t s}^{\mathrm{ESS} \text {,Pnet }}$ (the auxiliary variable defined in (16) and (19) characterizing the absolute value of $P_{t s}^{\mathrm{ESS}, \mathrm{Net}}$ ) [19]:

$$
\begin{gathered}
S O E_{t s}^{\mathrm{ESS}}=\mathrm{SOE}_{0}^{\mathrm{ESS}}+\frac{\tau_{1}}{3600} \sum_{t^{\prime}=1}^{t} \eta^{+}\left[\frac{\gamma_{t^{\prime} s}^{\mathrm{ESS}, \text { Pnet }}-P_{t^{\prime} s}^{\mathrm{ESS} \text { Net }}}{2}\right]- \\
-\frac{\tau_{1}}{3600} \sum_{t^{\prime}=1}^{t} \frac{1}{\eta^{-}}\left[\frac{\gamma_{t^{\prime} s}^{\mathrm{ESS} \text { Pnet }}+P_{t^{\prime} s}^{\mathrm{ESS}, \mathrm{Net}}}{2}\right] \quad \forall t \in \mathbb{T}, \forall s \in \mathbb{S} .
\end{gathered}
$$

It is noteworthy that the ramp rate limit of the RDGs'/ESS's converters are not required to be modeled in the problem due to the fact that the RDGs'/ESS's converters can move their power set-point from zero to their rated power limit in a couple of msec, while the method is solved with time resolution of seconds.

\section{Linear Scenario-Based Optimization Problem Formulation}

Thanks to the introduced equivalent linear optimization problems for all three terms of the objective function (4) and the presented framework for modeling the constraints of the grid, DERs and ESS, the first stage of the method can be formulated as a linear scenario-based optimization problem:

$$
\begin{aligned}
\min _{\xi^{\prime}} \pi_{0^{\prime}}^{\mathrm{Imb}, \mathrm{P}} \sum_{s \in \mathbb{S}} \sum_{t \in \mathbb{T}} \gamma_{0^{\prime} t s}^{\mathrm{Imb}, \mathrm{P}}+\pi_{0^{\prime}}^{\mathrm{Imb}, \mathrm{Q}} \sum_{s \in \mathbb{S}} \sum_{t \in \mathbb{T}} \gamma_{0^{\prime} t s}^{\mathrm{Imb}, \mathrm{Q}}+ \\
\pi^{\mathrm{ESS}, \mathrm{P}} \sum_{s \in \mathbb{S}} \sum_{t \in \mathbb{T}} \gamma_{t s}^{\mathrm{ESS}, \mathrm{P}}+\pi^{\mathrm{ESS}, \mathrm{SOE}} \gamma^{\mathrm{ESS}, \mathrm{SOE}}+\sum_{s \in \mathbb{S}} \sum_{t \in \mathbb{T}} \gamma_{t s}^{\mathrm{ESS}, \mathrm{Pnet}}+ \\
\quad+\sum_{k \in \mathbb{D D G}}\left[\gamma_{k}^{\mathrm{DDG}, \mathrm{P}+}+\gamma_{k}^{\mathrm{DDG}, \mathrm{P}-}\right],
\end{aligned}
$$

subject to (3), (6), (7), (10), (11), (15), (17)-(19), (20)-(22), (24), (25), (30)-(37), (39), (40)-(44). $\xi^{\prime}$ indicates the set of optimization variables consisting of $\xi$, introduced in (5), and the auxiliary variables as:

$\xi^{\prime}=\xi \cup\left\{P_{0^{\prime} t s}^{\mathrm{Imb}}, Q_{0^{\prime} t s}^{\mathrm{Imb}}, f_{k}^{\mathrm{DDG}, \mathrm{P}+}, f_{k}^{\mathrm{DDG}, \mathrm{P}-}, \gamma_{0^{\prime} t s}^{\mathrm{Imb}, \mathrm{P}}, \gamma_{0^{\prime} t s}^{\mathrm{Imb}, \mathrm{Q}}, \gamma_{t s}^{\mathrm{ESS}, \mathrm{P}}\right.$,
$\left.\left.\gamma^{\mathrm{ESS}, \mathrm{SOE}}, \gamma_{t s}^{\mathrm{ESS}, \mathrm{Pnet}}, \gamma_{k}^{\mathrm{DDG}, \mathrm{Pt}}, \gamma_{k}^{\mathrm{DDG}, \mathrm{P}-}\right\}, \quad, \quad 46\right)$

where operator $U$ calculates the union of two sets.

\section{SeCOND Stage: ESS REAL-Time CONTROL}

The second stage of the method starts at the beginning of the time slot and lasts until the end of the time slot, as illustrated in Fig. 2. This stage is designated to mitigate the impact of the mismatch between the forecasted consumption/generation of loads/RDGs and the realized ones on the active/reactive power imbalance at the PCC. Relying on a linear optimization problem, it controls the active/reactive power injections of the ESS to track the targeted active/reactive power flow at the PCC, i.e. $\mathrm{P}_{0^{\prime}}^{\text {Target }}$ and $\mathrm{Q}_{0^{\prime}}^{\text {Target }}$, while respecting the operational constraints of the ESS. The outlines of the control strategy are:

1- The whole time slot is split into $\mathrm{N}_{\kappa}$ time-intervals with duration $\tau_{2}$ and $\kappa$ is the index for time-intervals.

2- The reference active power set-point of ESS during each time-interval $\kappa$ can be retrieved from the value of $P^{\mathrm{ESS}, \text { Ref }}$ determined at the first stage:

$$
\widehat{\mathrm{P}}_{\kappa}^{\mathrm{ESS}, \mathrm{Ref}}=P^{\mathrm{ESS}, \mathrm{Ref}} \quad \kappa=1, \ldots, \mathrm{N}_{\kappa},
$$

3- The control strategy is executed at the beginning of each time-interval $\kappa$. The control action consists in determining and actuating the additional active and reactive powers flexibility, with respect to the reference power set-point $\widehat{\mathrm{P}}_{\kappa}^{\mathrm{ESS}, \mathrm{Ref}}$, that the ESS should provide during the current time-interval $\kappa$, i.e. $f_{\kappa}^{\mathrm{ESS}, \mathrm{P}}$ and $f_{\kappa}^{\mathrm{ESS}, \mathrm{Q}}$. They are constant values over the whole time-interval $\kappa$.

4- At the beginning of the time-interval $\kappa$, the most recent realized active/reactive powers flow at the PCC, i.e. $\mathrm{P}_{0^{\prime}(\kappa-1)}$ and $\mathrm{Q}_{0^{\prime}(\kappa-1)}$ are measured. Moreover, the actuated active/reactive powers of the ESS, i.e. $\widehat{\mathrm{P}}_{(\kappa-1)}^{\mathrm{ESS}, \mathrm{Ref}}+\mathrm{f}_{(\kappa-1)}^{\mathrm{ESS}, \mathrm{P}}$ and $\mathrm{f}_{(\kappa-1)}^{\mathrm{ESS}, \mathrm{Q}}$, during the previous timeinterval are known based on the outcome of the accomplished control over time-interval $\kappa-1$. Therefore, the net realized active/reactive power absorption of the ADN excluding ESS, i.e. $\mathrm{P}_{(k-1)}^{\mathrm{ADN}}$ and $\mathrm{Q}_{(\kappa-1)}^{\mathrm{ADN}}$ can be easily calculated as:

$\mathrm{P}_{(\kappa-1)}^{\mathrm{ADN}}=\mathrm{P}_{0^{\prime}(\kappa-1)}+\widehat{\mathrm{P}}_{(\kappa-1)}^{\mathrm{ESS}, \mathrm{Ref}}+\mathrm{f}_{(\kappa-1)}^{\mathrm{ESS}, \mathrm{P}}$

$\mathrm{Q}_{(\kappa-1)}^{\mathrm{ADN}}=\mathrm{Q}_{0^{\prime}(\kappa-1)}+\mathrm{f}_{(\kappa-1)}^{\mathrm{ESS}, \mathrm{Q}}$

whereby the net realized active/reactive power absorption of the ADN during the time-interval $\kappa$ is predicted to be equal to the one realized in the former 


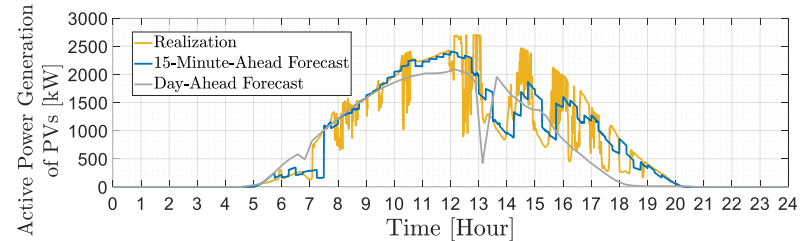

Fig. 6 Realization vs day-ahead/15-minute ahead forecast of the net active power generation of PVs.

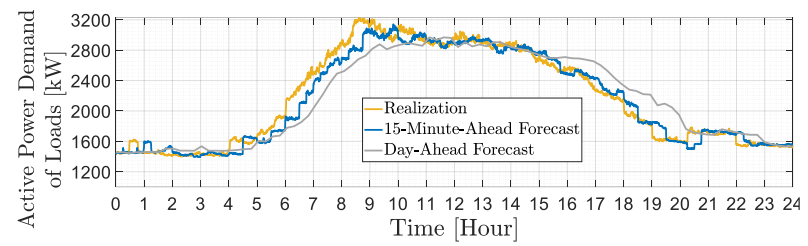

Fig. 7 Realization vs day-ahead/15-minute ahead forecast of the net active power demand of loads.
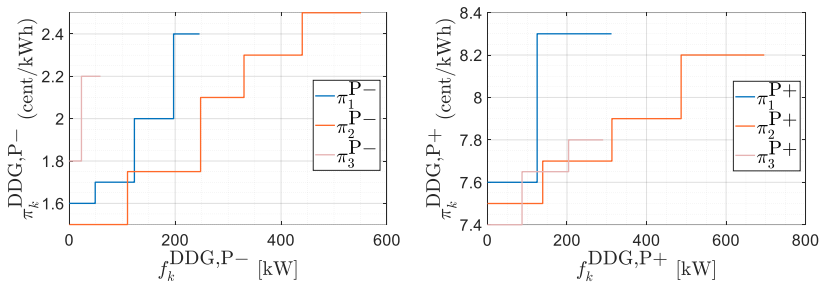

Fig. 8 Offer curve of DDGs for active power flexibility provision.

time-interval. Thus, the active/reactive power flow at PCC during the time-interval $\kappa$ is predicted to be:

$$
\begin{aligned}
P_{0^{\prime} \kappa} & =\mathrm{P}_{(\kappa-1)}^{\mathrm{ADN}}-\widehat{\mathrm{P}}_{\kappa}^{\mathrm{ESS}, \mathrm{Ref}}-f_{\kappa}^{\mathrm{ESS}, \mathrm{P}} \\
Q_{0^{\prime}{ }^{\prime}} & =\mathrm{Q}_{(\kappa-1)}^{\mathrm{ADN}}-f_{\kappa}^{\mathrm{ESS}, \mathrm{Q}}
\end{aligned}
$$

To mathematically formulate the control strategy, let us assume to be at the beginning of the time-interval $\kappa$. The control objective is to keep $P_{0^{\prime}{ }{ }^{\prime}}$ and $Q_{0^{\prime}{ }_{\kappa}}$ close to $\mathrm{P}_{0^{\prime}}^{\text {Target }}$ and $\mathrm{Q}_{0^{\prime}}^{\text {Target }}$, respectively. This control objective can be formulated as:

$$
\begin{aligned}
\min _{\psi_{\kappa}} \pi_{0^{\prime}}^{\mathrm{Imb}, \mathrm{P}} \mid P_{0^{\prime} \kappa}- & \mathrm{P}_{0^{\prime}}^{\text {Target }} \mid+ \\
& \pi_{0^{\prime}}^{\mathrm{Imb}, \mathrm{Q}}\left|Q_{0^{\prime} \kappa}-Q_{0^{\prime}}^{\text {Target }}\right|+\gamma_{\kappa}^{\text {ESS,Pnet }},
\end{aligned}
$$

where $\pi_{0^{\prime}}^{\mathrm{Imb}, \mathrm{P}}$ and $\pi_{0^{\prime}}^{\mathrm{Imb}, \mathrm{Q}}$ are weighting coefficients; the third term, i.e. $\gamma_{\kappa}^{\text {ESS,Pnet }}$, is a non-negative auxiliary variable defined to linearly model the evolution of the SOE over time-interval $\kappa$, as detailed in (54), (57) and (61); $\psi_{\kappa}$ indicates the set of control variables as:

$\psi_{\kappa}=\left\{f_{\kappa}^{\mathrm{ESS}, \mathrm{P}}, f_{\kappa}^{\mathrm{ESS}, \mathrm{Q}}\right\}$.

The nonlinear objective function (52) has an equivalent linear optimization problem as:

$\min _{\psi_{\kappa}} \pi_{0^{\prime}}^{\mathrm{Imb}, \mathrm{P}} \gamma_{\kappa}^{\mathrm{Imb}, \mathrm{P}}+\pi_{0^{\prime}}^{\mathrm{Imb}, \mathrm{Q}} \gamma_{\kappa}^{\mathrm{Imb}, \mathrm{Q}}+\gamma_{\kappa}^{\mathrm{ESS}, \text { Pnet }}$,

subject to

$-\gamma_{\kappa}^{\operatorname{Imb}, \mathrm{P}} \leq P_{0^{\prime} \kappa}-\mathrm{P}_{0^{\prime}}^{\mathrm{Target}} \leq \gamma_{\kappa}^{\mathrm{Imb}, \mathrm{P}}$

$-\gamma_{\kappa}^{\text {Imb,Q }} \leq Q_{0^{\prime}{ }^{\prime}}-\mathrm{Q}_{0^{\prime}}^{\text {Target }} \leq \gamma_{\kappa}^{\text {Imb, }, Q}$

$-\gamma_{\kappa}^{\text {ESS,Pnet }} \leq \widehat{\mathrm{P}}_{\kappa}^{\mathrm{ESS}, \mathrm{Ref}}+f_{\kappa}^{\mathrm{ESS}, \mathrm{P}} \leq \gamma_{\kappa}^{\mathrm{ESS}, \text { Pnet }}$

where $\gamma_{\kappa}^{\mathrm{Imb}, \mathrm{P}}, \gamma_{\kappa}^{\mathrm{Imb}, \mathrm{Q}}$ are non-negative auxiliary variables.

The power limit of the ESS can be expressed as:

$\left(\widehat{\mathrm{P}}_{\kappa}^{\mathrm{ESS}, \mathrm{Ref}}+f_{\kappa}^{\mathrm{ESS}, \mathrm{P}}\right)^{2}+f_{\kappa}^{\mathrm{ESS}, \mathrm{Q}^{2}} \leq \mathrm{S}^{\mathrm{ESS}, \mathrm{Max}^{2}}$

where $S^{\text {ESS,Max }}$ is the ESS's rated power limit. Similar to the approach adopted for linearizing constraint (38) and depicted in Fig. 4, the nonlinear constraint (58) can be expressed as a set of linear constraints with constant coefficients $\mathbf{E}_{m}^{0}, \mathbf{E}_{m}^{\mathrm{P}}$ and $\mathbf{E}_{m}^{\mathrm{Q}}$ as:

$\mathbf{E}_{m}^{0}+\mathbf{E}_{m}^{\mathrm{P}}\left(\widehat{\mathrm{P}}_{\kappa}^{\mathrm{ESS}, \mathrm{Ref}}+f_{\kappa}^{\mathrm{ESS}, \mathrm{P}}\right)+\mathbf{E}_{m}^{\mathrm{Q}} f_{\kappa}^{\mathrm{ESS}, \mathrm{Q}} \leq 0 \quad \forall m \in \mathbb{A}_{e}$ where $\mathbb{A}_{e}$ is the set of linear constraints modeling the nonlinear maximum power constraint of the ESS, $m$ is the index for the linear constraints belonging to $\mathbb{A}_{e}$.

The energy limits of the ESS can be modeled as: $\mathrm{SOE}^{\mathrm{ESS}, \mathrm{Min}} \leq S O E_{\kappa}^{\mathrm{ESS}} \leq \mathrm{SOE}^{\mathrm{ESS}, \mathrm{Max}}$

The evolution of $S O E_{\kappa}^{\mathrm{ESS}}$ over time-interval $\kappa$ can be expressed as a linear function of $\widehat{\mathrm{P}}_{\kappa}^{\mathrm{ESS}, \mathrm{Ref}}, f_{\kappa}^{\mathrm{ESS}, \mathrm{P}}$ and $\gamma_{\kappa}^{\mathrm{ESS}, \mathrm{Pnet}}$ (the auxiliary variable defined in (52), (54) and (57) characterizing the absolute value of $\widehat{\mathrm{P}}_{\kappa}^{\mathrm{ESS}, \mathrm{Ref}}+f_{\kappa}^{\mathrm{ESS}, \mathrm{P}}$ ) [19]:

$$
\begin{aligned}
S O E_{\kappa}^{\mathrm{ESS}}= & \mathrm{SOE}_{\kappa-1}^{\mathrm{ESS}}+\frac{\tau_{2}}{3600} \eta^{+}\left[\frac{\gamma_{\kappa}^{\mathrm{ESS}, \mathrm{Pnet}}-\widehat{\mathrm{P}}_{\kappa}^{\mathrm{ESS}, \mathrm{Ref}}-f_{\kappa}^{\mathrm{ESS}, \mathrm{P}}}{2}\right] \\
& -\frac{\tau_{2}}{3600} \frac{1}{\eta^{-}}\left[\frac{\left.\gamma_{\kappa}^{\mathrm{ESS}, \mathrm{Pnet}}+\widehat{\mathrm{P}}_{\kappa}^{\mathrm{ESS}, \mathrm{Ref}}+f_{\kappa}^{\mathrm{ESS}, \mathrm{P}}\right]}{2}\right]
\end{aligned}
$$

The objective function (54) subject to (50), (51), (55)-(57), (59)-(61) forms a linear optimization problem whose the solution determines the final power set-point of the ESS over the time-interval $\kappa$.

\section{CAse Study And Results}

The performance of the method is validated considering the real distribution network shown in Fig. 1, which is located in the city of Aigle, in southwest of Switzerland. It includes 55 buses at $21 \mathrm{kV}$ accommodating $2700 \mathrm{kWp}$ installed solar PV units (RDGs), $2150 \mathrm{~kW}$ installed hydro-power units (DDGs) and a $1000 \mathrm{kVA} / 500 \mathrm{kWh}$ utility-scale Lithium Titanate ESS with charging (discharging) efficiency of $94 \%(96 \%)$. In line with the timeline of the problem detailed in Fig. $2, \mathrm{t}_{0}, \mathrm{t}_{\mathrm{ADN}}, \mathrm{T}$, $\tau_{1}$ and $\tau_{2}$ are considered 15 minutes, 15 minutes, 15 minutes, 30 seconds, 1 second respectively. A particular day where the solar irradiance is very volatile is considered as 24 hours of study. Fig. 6 and Fig. 7 respectively show the net active power generation/consumption of $\mathrm{PVs} / \mathrm{loads}$ throughout the day (realization vs forecasts). The k-nearest neighbors algorithm [20] is exploited to carry out the 15-minute ahead forecasts and to generate the 1000 scenarios required in the first stage of the method, i.e. $s \in \mathbb{S}$. The ESS's SOE at the beginning of the day is set to $250 \mathrm{kWh}$ and the active/reactive powers flexibility request of the upper-layer grid, $\hat{\mathrm{f}}_{0^{\prime}}^{\mathrm{P}}$ and $\hat{\mathrm{f}}_{0^{\prime}}^{\mathrm{Q}}$, are respectively set equal to $-400 \mathrm{~kW}^{1}$ and $0 \mathrm{kVAr}$ throughout the day. Minimum and maximum of the nodal voltage magnitude limits are chosen as 0.95 p.u. and 1.05 p.u. The objective function's weighting coefficients $\pi_{0^{\prime}}^{\mathrm{Imb}, \mathrm{P}}, \pi_{0^{\prime}}^{\mathrm{Imb}, \mathrm{Q}}, \pi^{\mathrm{ESS}, \mathrm{P}}$ and $\pi^{\mathrm{ESS}, \mathrm{SOE}}$ are respectively assumed $100 \mathrm{cent} / \mathrm{kW}, 50 \mathrm{cent} / \mathrm{kVAr}, 30 \mathrm{cent} / \mathrm{kW}, 10 \mathrm{cent} / \mathrm{kW}$, to prioritize different terms of the objective function for deploying the available local flexibility. The offer curves of DDGs are shown in Fig. 8, where indices 1, 2 and 3 refer to the DDGs connected to nodes 11,53 and 55 . Then, the problem is modeled by using YALMIP-MATLAB [21] and solved with 


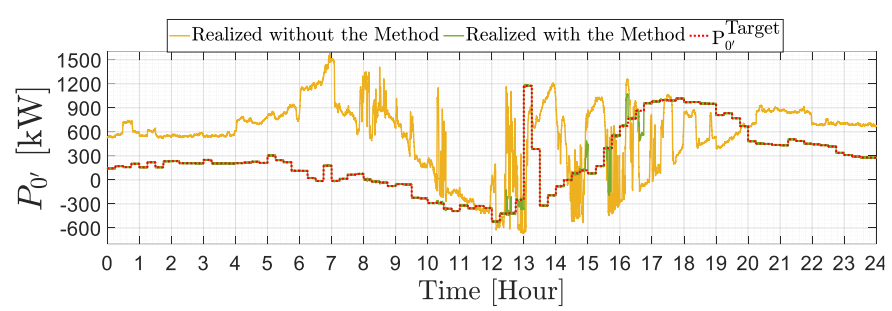

Fig. 9 Realized active power flow at the PCC.

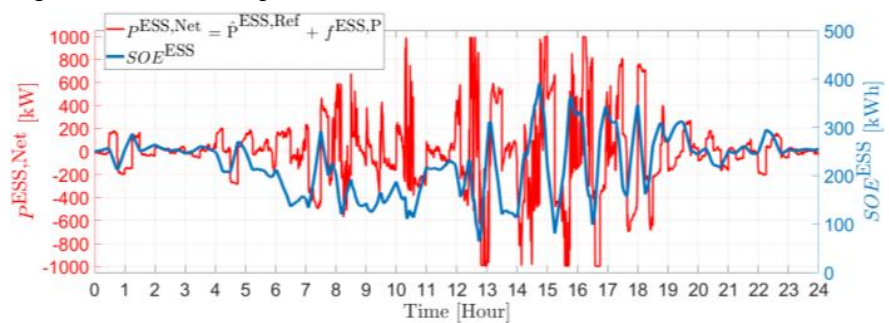

Fig. 10 Evolution of the ESS's SOE/net power injection throughout the day.

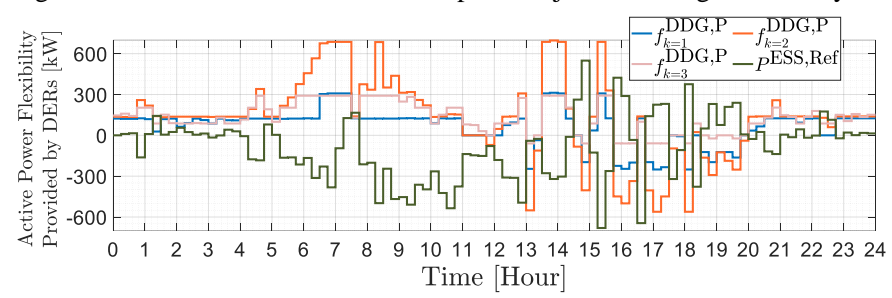

Fig. 11 Provided active power flexibility of the DDGs along with the determined reference active power set-point of the ESS.

GUROBI solver [22] on a Windows based system with a 2.8 $\mathrm{GHz}$ Xeon CPU and 16 GB RAM.

Fig. 9 shows the profiles of the targeted active power operating point, i.e. $\mathrm{P}_{0^{\prime}}^{\text {Target }}$, (red curve) along with the realized active power flow at the PCC before (orange curve) and after (green curve) applying the method. For the sake of brevity, lets introduce difference as difference between the red and orange curves. difference is composed of 1-the requested power flexibility of the upper-layer grid, plus 2-the day-ahead forecast errors of demand and renewable generation. Due to the absence of solar irradiance, over periods (00:00 to 05:00) and (20:00 to 24:00) uncertainties result solely from the forecast errors of demand leading to moderate day-ahead and 15-minute-ahead forecast errors with (average, maximum) of $(60 \mathrm{~kW}, 287 \mathrm{~kW})$ and $(54 \mathrm{~kW}, 224 \mathrm{~kW})$, respectively. Consequently, difference over these two periods embraces small volatility and mainly reflects the requested power flexibility at the PCC, thus, the ESS can accomplish its task, i.e. mitigating the impact of the 15-minute-ahead forecast errors on the imbalance at the PCC, by taking up a marginal activity as shown in Fig. 10. As result of this marginal activity, the ESS's SOE remains near to the middle, i.e. $250 \mathrm{kWh}$, whereby the reference active power setpoint of the ESS ( $P^{\mathrm{ESS}, \mathrm{Ref}}$ ) is set equal to zero over most of these two periods as shown in Fig. 11. Furthermore, the power flexibility of DERs is mainly procured to cover the requested power flexibility of the upper-layer grid, as shown in Fig. 11. In contrast, over period (05:00 to 20:00) the uncertainties, in addition to the moderate forecast error of demand, contain extremely volatile forecast error of PVs leading to day-ahead and 15-minute-ahead forecast errors with (average, maximum) of $(544 \mathrm{~kW}, 2238 \mathrm{~kW})$ and $(292 \mathrm{~kW}, 1435 \mathrm{~kW})$, respectively.

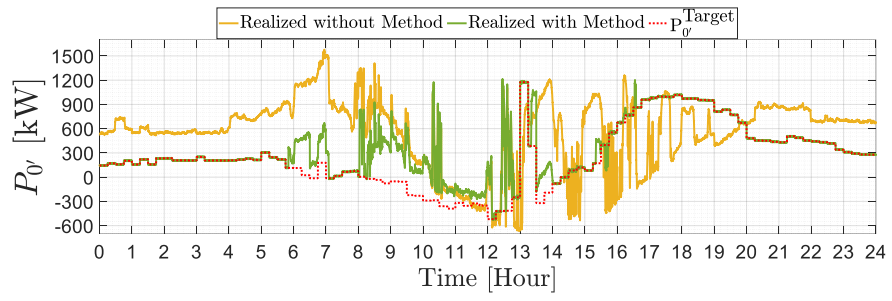

Fig. 12. Realized active power flow at the PCC (for the case where $P^{\mathrm{ESS} \text {, Ref }}$ is not embedded in the first stage).

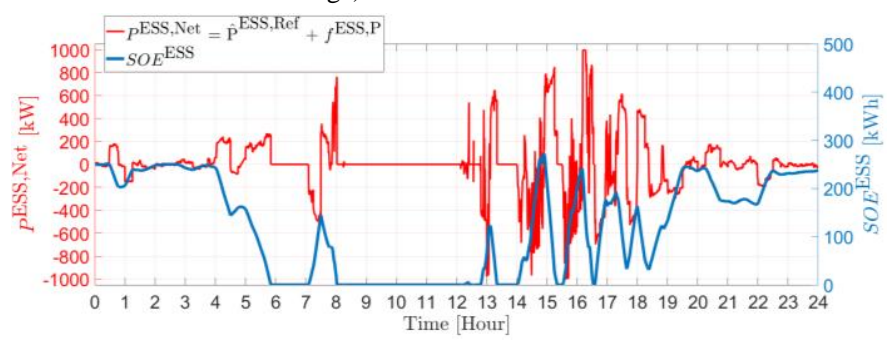

Fig. 13. Evolution of the ESS's SOE/net power injection throughout the day (for the case where $P^{\mathrm{ESS}, \mathrm{Ref}}$ is not embedded in the first stage).

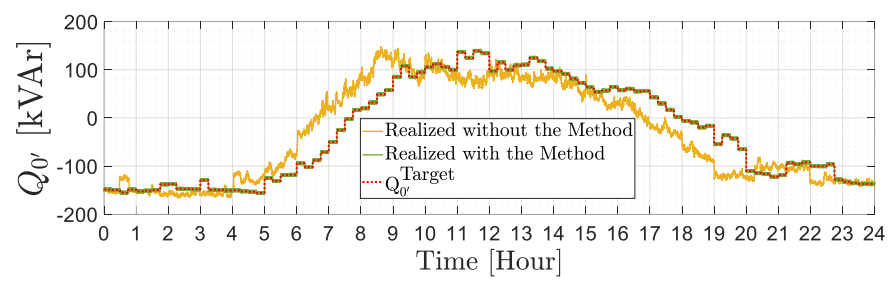

Fig. 14 Realized reactive power flow at the PCC.

Consequently, difference over this period features an extreme volatility and the ESS plays a crucial role to mitigate the impact of the 15-minute-ahead forecast error on the imbalance at the PCC while restoring its SOE to the middle. Fig. 11 illustrates how the active power flexibility of DERs are procured for the purpose of 1-satisfying the requested active power flexibility of the upper-layer grid as well as 2-helping the ESS to restore its SOE to the middle.

In spite of these large and volatile uncertainties, the ESS succeeded to track the target, i.e. $\mathrm{P}_{0^{\prime}}^{\text {Target }}$, with average accuracy of $98.14 \%$ throughout 24 hours of study. This quality of result is achieved thanks to the appropriate decision made in the first stage, i.e. $P^{\mathrm{ESS}, \mathrm{Ref}}$ which is exclusively designated to manage the ESS's SOE. However, in total, during 41 minutes and 53 seconds (cumulated time) of the day, the ESS cannot thoroughly track $\mathrm{P}_{0^{\prime}}^{\text {Target }}$ due to the fact that the 15-minuteahead forecast error of PVs is larger than the rated power limit of the ESS, i.e. $1000 \mathrm{~kW}$. In this respect, Fig. 10 shows the ESS reaching its maximum rated power limit in multiple times, but it never reaches its energy limits thanks to $P^{\mathrm{ESS}, \mathrm{Ref}}$. It is noteworthy that if $P^{\mathrm{ESS} \text {, Ref }}$ was not embedded in the first stage i.e. $P^{\mathrm{ESS}, \mathrm{Ref}}=0$, as shown in Fig. 12, the ESS could not thoroughly track $\mathrm{P}_{0^{\prime}}^{\text {Target }}$ over 6 hours and 40 minutes and 16 seconds of the day due to reaching either its energy or its power limits as shown in Fig. 13.

The method is able to track $Q_{0^{\prime}}^{\text {Target }}$ with average accuracy of 99.99\% throughout the day, as shown in Fig. 14. Finally, it is worth noting that the computation time of the first stage (for each time slot) and the second stage (for each time-interval) of 
the method are $8.79 \mathrm{sec}$ and $3.1 \mathrm{msec}$, respectively.

\section{CONCLUSIONS}

To help unlocking the potential power flexibilities available in ADNs, this paper proposes a two-stage DERs/ESS coordination and control method to provide power flexibility at the ADN's PCC. Based on the active/reactive powers flexibility request of the upper-layer grid operator, it firstly relies on a linear scenario-based optimization problem to determine the optimal amount of the power flexibility that each DER should provide. To this end, it considers offer curve of DERs, uncertainties of demand and renewable generation as well as operational constraints of the grid and DERs/ESS. Then, during the real-time operation, it exploits a linear optimization formulation to control the active/reactive power injection of a utility-scale ESS to precisely track the requested power flexibility at the ADN's PCC while counteracting the impact of the day-ahead forecast errors. A real distribution network located in the city of Aigle in southwest of Switzerland is used to validate the performance of the proposed method. The results show that the method is able to precisely satisfy the flexibility request of the upper-layer grid operator at the PCC, while successfully managing the ESS's SOE.

\section{ACKNOWLEDGEMENTS}

The authors gratefully acknowledge the financial support from Swissgrid (the swiss transmission grid operator (TSO)) and the Swiss Innovation Agency. This work was carried out within the frame of the Swiss Centre for Competence in Energy Research on the Future Swiss Electrical Infrastructure (SCCERFURIES).

\section{REFERENCES}

[1] N. M. Haegel, R. Margolis, T. Buonassisi, D. Feldman, A. Froitzheim, R. Garabedian, M. Green, S. Glunz, H. M. Henning, B. Holder, and I. Kaizuka, "Terawatt-scale photovoltaics: Trajectories and challenges," Science, vol. 356, no. 6334 , pp. 141-143, Apr. 2017.

[2] S. Xia, Z. Ding, T. Du, D. Zhang, M. Shahidehpour, and T. Ding, "Multitime Scale Coordinated Scheduling for the Combined System of Wind Power, Photovoltaic, Thermal Generator, Hydro Pumped Storage, and Batteries," IEEE Transactions on Industry Applications, vol. 56, no. 3, pp. 2227-2237, May 2020.

[3] X. B. Wang, J. X. Chang, X. J. Meng, and Y. M. Wang, "Short-term hydrothermal-wind-photovoltaic complementary operation of interconnected power systems," Appl. Energy, vol. 229, pp. 945-962, 2018.

[4] R. Doherty, and M O'malley, "A new approach to quantify reserve demand in systems with significant installed wind capacity," IEEE Transaction on Power Systems, volume 20, number 2, 2005.

[5] S. R. Salkuti, "Day-ahead thermal and renewable power generation scheduling considering uncertainty," Renew. Energy, vol. 131, no. 1, pp. 956 965,2019

[6] L. Mehigan, J. P. Deane, B. P. Ó. Gallachóir, and V. Bertsch, "A review of the role of distributed generation (DG) in future electricity systems," Energy, vol. 163, pp. 822-836, Nov. 2018.

[7] K. Christakou, D.-C. Tomozei, J.-Y. Le Boudec, and M. Paolone, "GECN: Primary Voltage Control for Active Distribution Networks via Real-Time Demand-Response," IEEE Transactions on Smart Grid, vol. 5, no. 2, pp. 622 631, Mar. 2014.

[8] A. Salazar, A. Berzoy, W. Song, and J. M. Velni, "Energy Management of Islanded Nanogrids Through Nonlinear Optimization Using Stochastic Dynamic Programming," IEEE Transactions on Industry Applications, vol. 56, no. 3, pp. 2129-2137, May 2020.
[9] M. J. Dolan, E. M. Davidson, I. Kockar, G. W. Ault, and S. D. J. McArthur, "Distribution Power Flow Management Utilizing an Online Optimal Power Flow Technique," IEEE Transactions on Power Systems, vol. 27, no. 2, pp. 790-799, May 2012.

[10] L. Zhao, W. Zhang, H. Hao, and K. Kalsi, "A Geometric Approach to Aggregate Flexibility Modeling of Thermostatically Controlled Loads," IEEE Transactions on Power Systems, vol. 32, no. 6, pp. 4721-4731, Nov. 2017.

[11] S. Gill, I. Kockar, and G. W. Ault, "Dynamic Optimal Power Flow for Active Distribution Networks," IEEE Transactions on Power Systems, vol. 29, no. 1, pp. 121-131, Jan. 2014.

[12] A. Saint-Pierre and P. Mancarella, "Active Distribution System Management: A Dual-Horizon Scheduling Framework for DSO/TSO Interface Under Uncertainty," IEEE Transactions on Smart Grid, vol. 8, no. 5, pp. 21862197, Sep. 2017.

[13] T. Ding, C. Li, Y. Yang, J. Jiang, Z. Bie, and F. Blaabjerg, “A Two-Stage Robust Optimization for Centralized-Optimal Dispatch of Photovoltaic Inverters in Active Distribution Networks," IEEE Transactions on Sustainable Energy, vol. 8, no. 2, pp. 744-754, Apr. 2017.

[14] G. Migliavacca, Ed., TSO-DSO Interactions and Ancillary Services in Electricity Transmission and Distribution Networks: Modeling, Analysis and Case-Studies. Springer International Publishing, 2020.

[15] M. Kalantar-Neyestanaki, F. Sossan, M. Bozorg, and R. Cherkaoui, "Characterizing the Reserve Provision Capability Area of Active Distribution Networks: A Linear Robust Optimization Method," IEEE Transactions on Smart Grid, vol. 11, no. 3, pp. 2464-2475, May 2020.

[16] F. Capitanescu, "TSO-DSO interaction: Active distribution network power chart for TSO ancillary services provision," Electric Power Systems Research, vol. 163, pp. 226-230, Oct. 2018.

[17] M. Kalantar-Neyestanaki, M. Bozorg, F. Sossan, and R. Cherkaoui, "Allocation of Frequency Control Reserve from Aggregated Resources of Active Distribution Systems," in 2018 Power Systems Computation Conference (PSCC), Jun. 2018.

[18] A. Bernstein and E. Dall'Anese, "Linear power-flow models in multiphase distribution networks," in 2017 IEEE PES Innovative Smart Grid Technologies Conference Europe (ISGT-Europe), Sep. 2017.

[19] S. P. Boyd and L. Vandenberghe, Convex optimization. Cambridge, UK; New York: Cambridge University Press, 2004.

[20] Y. Chu and C. F. M. Coimbra, "Short-term probabilistic forecasts for Direct Normal Irradiance," Renewable Energy, vol. 101, pp. 526-536, Feb. 2017.

[21] J. Löfberg, "YALMIP: A toolbox for modeling and optimization in MATLAB," in Proc. CACSD Conf., Taipei, Taiwan, pp. 284-289, 2004.

[22] "Gurobi Optimizer Reference Manual," Gurobi Optimization, Inc. [Online]. Available: http://www.gurobi.com.

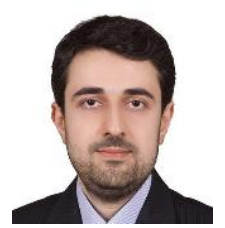

Mohsen Kalantar-Neyestanaki (S'14) received the B.Sc. degrees (Hons.) in electrical engineering and physics, and the M.Sc. degree (Hons.) in electrical engineering from Sharif University of Technology, Tehran, Iran, in 2011 and 2013, respectively, where he worked as a senior researcher with the Power Systems Research Group from 2013 to 2015. From 2015 to 2016, he worked as a R\&D engineer in the Wide Area Measurement System (WAMS) control center of Iran Grid Management Co. (the TSO of Iran), Tehran, Iran. Since 2017, he has been pursuing the Ph.D. degree with the Power Systems Group at Ecole Polytechnique Fédérale de Lausanne (EPFL), Lausanne, Switzerland. His research interests include modernizing power systems with particular reference to the integration of distributed storage and energy resources; numerical optimization and modeling techniques for power systems control, operation and planning; and wide-area monitoring, protection and control systems.

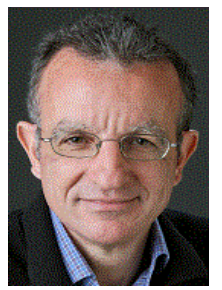

Rachid Cherkaoui (M'05-SM'07) received both the M.Sc. and Ph.D. degrees in electrical engineering in 1983 and 1992, respectively, from the Swiss Federal Institute of Technology in Lausanne (EPFL), Switzerland. He is currently Senior scientist at EPFL, leading the power systems group. His research interests are in electricity market deregulation, distributed generation and storage, and power system vulnerability mitigation.

$\mathrm{He}$ is member of technical program committees of various conferences and was member of CIGRE TF's and WG's. He was IEEE Swiss chapter officer from 2005 to 2011 . He is author and co-author of more than 100 scientific publications. 